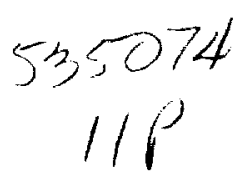

A IA A-2000-4322

\title{
COMPUTATIONAL MODELING FOR THE TRANSITIONAL FLOW OVER A MULTI-ELEMENT AIRFOIL
}

\author{
William W. Liou* and Fengjun Liu** \\ Department of Mechanical and Aeronautical Engineering \\ Western Michigan University \\ Kalamazoo, MI 49008
}

\begin{abstract}
The transitional flow over a multi-element airfoil in a landing configuration are computed using a twoequation transition model. The transition model is predictive in the sense that the transition onset is a result of the calculation and no prior knowledge of the transition location is required. The computations were performed using the INS2D Navier-Stokes code. Overset grids are used for the three element airfoil. The airfoil operating conditions are varied for a range of angle of attack and for two different Reynolds numbers of 5 million and 9 million. The computed results are compared with experimental data for the surface pressure, skin friction, transition onset location, and velocity magnitude. In general, the comparison shows a good agreement with the experimental data.
\end{abstract}

\section{INTRODUCTION}

Flows past multi-element airfoils have been subjected to intensive experimental and computational studies ${ }^{1,2,3}$ for the past two decades. An accurate prediction of such flows can enhance the performance and the safety factor of aircrafts in high-lift operations. Even with the advances of computational fluid dynamics (CFD), the prediction of high-lift flow fields remains a challenge. The flow fields around multi-element airfoils are complex and are known to be dominated by different flow mechanisms at different operating conditions.

A significant portion of the flow around multielement airfoils is transitional over a wide range of operating condition. The onset location of the transition process varies with, for example, angles of attack and Reynolds numbers. In addition to boundary layer

\footnotetext{
* Associate Professor, Senior Member AIAA.

** Graduate Assistant.

Copyright(C)2000 by the authors. Published by the American Institute of Aeronautics and Astronautics, Inc, with permission.
}

transition, free shear flow transition is also likely to occur in, for example, the flow over the slat. As the slat wake convects downstream, it may further interact with the transitional flow over the succeeding elements, such as the flow over the main element and the flap. As a result, an accurate prediction of the flow transition represents a major challenge for the computation of the flow over multi-element airfoils and the use of a transition model often becomes a discriminating factor in the outcomes of a prediction. A comprehensive evaluation of existing transitional empirical correlations ${ }^{4}$ showed that none of the correlations evaluated gave satisfactory results for the rather complex high lift flows.

Turbulence models are frequently used in the highlift flow calculations. In this practice, turbulence models are "turned-on" or "ramped-up" at a designated location on the airfoils. The designated locations are usually correlated with the measured location of transition onset and the computational results can vary significantly with the assigned transition location.

In this paper, the transitional flow fields over a multi-element airfoil are calculated using a $k-\varepsilon$ twoequation transition model ${ }^{5}$. The model uses an effective eddy-viscosity by coupling an intermittence-like corrections to a turbulence eddy-viscosity. The intermittence function varies with the evolution of the disturbance kinetic energy, $k$, which is a solution variable for the present $k-\varepsilon$ modeling methodology. As a result, there is no need for any prior knowledge of the targeted transition process. In this sense, the present model is a truly predictive transition model. The model has been applied successfully to by-pass transitional flows over flat plates ${ }^{5}$. The predicted transition onset and the length of the transition region were in a very good agreement with the measurement for all the cases studied. The transition model and the results of its application to a multi-element airfoil are presented in the following sections.

The Navier-Stokes solver used in the study is described in the next section. The Chimera composite grids used are also presented. 


\section{FLOW SOLVER}

The flow calculations presented here were performed with the INS2D code $e^{6,7,8}$. The code solves the twodimensional, incompressible form of the Reynoldsaveraged Navier-Stokes equations using an artificial compressibility approach. The INS2D code is a finite difference solver, using Roe's third order upwindbiased, flux-difference splitting for the convective terms and a second-order central differencing for the viscous terms. The code is capable of solving both steady and unsteady problems with point-to-point matched grids or Chimera overset grids. The details of the INS2D code can be found in the references cited above. The INS2D code has been applied extensively to the flow over various single- and multi-element airfoils $6,7,8$ and has been shown to be a very reliable tool for such purposes. Generating the necessary grids for multielement airfoil flow calculations can be very timeconsuming regardless of the grid topology one chooses to use. In this study, overset grids were used and the grids were generated using the OVERMAGG ${ }^{9}$ software. OVERMAGG is an automated script system specifically designed to perform overset grid generation for multi-element airfoils. The use of OVERMAGG has resulted in a significant amount of saving in time during this study.

The present transition model was implemented as a separate module to the INS2D code. There are three other model options in the INS2D code. These models have been applied to the flow over the $30 \mathrm{P} 30 \mathrm{~N}$ airfoil geometry which is also used in this study and the results have been reported ${ }^{3}$.

\section{TRANSITION MODEL}

The transitional model is developed based on the experimental observation that the flows in transition are highly intermittent. Locally, the flow is turbulent as the turbulent spots convect through. In between the passing of the turbulent spots, the flow relaxes to a disturbed laminar state. In this case, a measure of the fraction of time that the boundary layer is turbulent is the intermittency factor. Normally, the intermittent nature of the transitional flow is not accounted for in the development of turbulence models. Turbulence models have been in general designed for flows in a fully turbulent state. To be able to use a turbulence model in the intermittent transitional flow region, we incorporate a weighting factor, $\gamma$, in the turbulent eddy viscosity , $\mu_{t}$, obtained from a "parent" two-equation turbulence model. That is,

$$
\mu_{t r}=\gamma \mu_{t}
$$

where $\mu_{t r}$ denotes transitional flow eddy-viscosity. The $\gamma$ function, or intermittency correction function, resembles the physical flow intermittency factor. Similar to the intermittency factor that determines the fraction of time the flow is turbulent in the transition region, $\gamma$ determines the fraction of turbulent eddy-viscosity to be used in the transition region. Therefore, $\gamma$ should also vary monotonically through the transition region.

In the present study, the intermittency correction function $\gamma$ is defined in terms of the streamwise variation of the peak disturbance energy. The disturbance kinetic energy increases as the flow evolves from laminar to transitional state. Its local peak level also increases.

Therefore, the disturbance energy level is an appropriate parameter to characterize the flow transitional region. The intermittency correction function involves the peak disturbance energy level and the local free stream turbulence level.

$$
\begin{gathered}
\gamma=\gamma_{0}+\left(1-\gamma_{0}\right) f^{\frac{2}{5}} \\
f=\frac{k_{n}^{+}-k_{1}^{+}}{k_{2}^{+}-k_{1}^{+}}
\end{gathered}
$$

where

$$
\begin{gathered}
k_{n}^{+} \equiv k_{n}^{+}(x)=\left.\max \left(k^{+}(x, y)\right)\right|_{x}-k_{e}^{+}(x) \\
k_{e}^{+}(x)=\frac{k_{e}(x)}{u_{\tau}^{2}(x)}
\end{gathered}
$$

$k_{e}$ and $u_{\tau}$ denote the local disturbance kinetic energy in the free stream and the local frictional velocity. The free stream turbulence intensity was quite low in the wind tunnel where the transition measurement were made. In the present application, $k_{e}$ is set according to the turbulence level in the outer stream. The model coefficient $k_{1}^{+}$relates to the threshold for the formation of turbulent spots near the wall. It has been shown ${ }^{10}$ that turbulent energetic eddies reduce to Kolmogorov eddies near the wall, where $k^{+}=0.25$, and all the wall parameter are characterized by Kolmogorov microscales. An estimate for the spot generation threshold level in terms of $k^{+}$is thus set at 0.25 . It should be noted that the wall boundary condition for $k$ was also determined by the Kolmogorov behavior of near-wall turbulence in the parent two-equation model used in this study. Therefore, the selection of the value of $k_{1}^{+}$is consistent with the current parent model. $k_{2}^{+}$is determined by examining fully developed turbulent boundary layer and its value is set at 4.5 . The term $k_{2}^{+}-k_{1}^{+}$in Eq.(3) is used 
to normalize the term $k_{n}^{+}-k_{1}^{+}$, such that the transition progress variable, $f$, satisfies the equation,

$$
0 \leq f \leq 1
$$

The effect of the local free stream turbulence is accounted for in Eqs.(4) and (5). In Eq.(4), $k_{n}^{+}$represents the difference between the peak value and the free stream value of the $k^{+}$profile at a streamwise location, $x$. In this study, the $k^{+}$profile is taken along the direction normal to the surface. Eq.(4) allows the flow transition to proceed according to the development of the internal peak level of $k^{+}$.

As was mentioned earlier, the present transition model coupled the intermittency formulation with a parent two-equation turbulence model. The parent model used here is a variation of the Shih and Lumley model ${ }^{10}$ and has been used in Liou and Shih ${ }^{11}$ and Liou et. $a^{12}$.

The model equations for $k$ and $\epsilon$ are,

$$
\begin{aligned}
k_{, t}+U_{i} k_{, i} & =\left[\left(\nu+\nu_{t}\right) k_{, i}\right]_{, i}-\overline{u_{i} u_{j}} U_{i, j}-\varepsilon \\
\varepsilon_{, i}+U_{i} \varepsilon_{, i} & =\left[\left(\nu+\frac{\nu_{t}}{\sigma_{\varepsilon}}\right) \varepsilon_{, i}\right]_{, i}-C_{1} \frac{\varepsilon}{k} \overline{u_{i} u_{j}} U_{i, j} \\
& -C_{2} f_{2} \frac{\varepsilon^{2}}{k}+\nu \nu_{t} S_{, i} S_{, i}
\end{aligned}
$$

where

$$
\begin{gathered}
C_{1}=1.44, \quad C_{2}=1.92, \quad \sigma_{\varepsilon}=1.3 \\
f_{2}=1-0.22 \exp \left[-\left(\frac{R_{t}}{6}\right)^{2}\right], \quad R_{\mathrm{t}}=\frac{k^{2}}{\nu \varepsilon} \\
C_{\mu}=\frac{1}{A_{0}+A_{s} U^{(*)} \frac{k}{\epsilon}}
\end{gathered}
$$

where

$$
\begin{aligned}
U^{(*)} & =\sqrt{S_{i j} S_{i j}+\bar{\Omega}_{i j} \bar{\Omega}_{i j}} \\
\bar{\Omega}_{i j} & =\tilde{\Omega}_{i j}-2 \epsilon_{i j k} \omega_{k} \\
\tilde{\Omega}_{i j} & =\Omega_{i j}-\epsilon_{i j k} \omega_{k}
\end{aligned}
$$

$\Omega_{i j}$ is the mean rotation rate viewed in a rotating reference frame with the angular velocity $\omega_{k}$. The parameter $A_{s}$ is determined by

$$
\begin{aligned}
A_{s} & =\sqrt{6} \cos \phi, \quad \phi=\frac{1}{3} \arccos (\sqrt{6} W) \\
W & =\frac{S_{i j} S_{j k} S_{k i}}{\bar{S}^{3}} \\
\bar{S} & =\sqrt{S_{i j} S_{i j}}, \quad S=\sqrt{2 S_{i j} S_{i j}}
\end{aligned}
$$

The damping function is defined by

$$
f_{\mu}=\left[1 .-\exp \left(-\left(a_{1} R_{k}+a_{3} R_{k}^{3}+a_{5} R_{k}^{5}\right)\right)\right]^{\frac{1}{2}}
$$

where

$$
\begin{gathered}
a_{1}=1.5 \times 10^{-3}, \quad a_{3}=10^{-9}, a_{5}=5 \times 10^{-10} \\
R_{k}=\frac{\sqrt{k} y}{\nu}
\end{gathered}
$$

The near-wall boundary conditions for the turbulent quantities are

$$
k=0.25 u_{\tau}^{2} \text { and } \varepsilon=0.251 \frac{u_{\tau}^{4}}{\nu}
$$

where $u_{\tau}$ denotes the surface friction velocity.

Note that no measurement was made to determine the turbulent intensity and the decay of turbulent kinetic energy in the experiment where the present data were taken. These information are needed to determine the value of the disturbance kinetic energy and its dissipation rate at the inlet of the computational domain in the present approach. A turbulent intensity level of $0.05 \%$ was cited and used in the present analysis and the equivalent eddy-viscosity was set at the laminar level.

The present intermittency correction function technique uses the variation of the disturbance energy to computationally characterize the transition progress. Therefore, the calculated intermittency correction function does not necessarily correspond to the physical flow intermittency factor.

\section{GEOMETRY}

The McDonnell Douglas 30P-30N landing configuration was used in this study. Figure 1 shows the geometry and the stations where profile data will be presented. Both the leading-edge slat and the trailingedge flap have a deflection angle of $30^{\circ}$. The airfoil has been tested extensively at NASA Langley LowTurbulence Pressure Tunnel (LTPT) ${ }^{1,13}$. The geometry has been used as a test case in a CFD Challenge Workshop held at NASA Langley in 1993 and many computational results obtained by using different solvers and models have been reported ${ }^{2,3,8}$.

The computational grid consists of seven zones and a total of about 103,000 grid points. Figure 2 shows the $\mathrm{C}$ grids around the slat, the main element, and the flap, which consist of $175 \times 45,381 \times 133$, and $221 \times 85$ points, respectively. Two $\mathrm{H}$ grids were used for the main-element cove and for the wake region of the flap. A background $\mathrm{H}$ grid extends to the top and the bottom walls of the wind tunnel. When the grid for one element overlaps another element(s), holes and outer boundaries are determined by using the PEGSUS code. 
The communication between the overset grids are also handled by the PEGSUS code.

Comprehensive grid-independence studies have been performed and reported previously using similar overset grids for the same geometry ${ }^{3,8}$. These and other calculations for the same geometry indicate that the grid point distributions in the wake regions can have certain effects on the velocity profiles in those regions and the wall pressure is not sensitive to the grid density. These results also show that further refinement of the grid from the current level does not change the results appreciably.

In the following, results will be presented for two Reynolds numbers of five million and nine million and a range of angle of attack (AOA) for each Reynolds number.

\section{RESULTS AND DISCUSSIONS}

Figure 3 shows a comparison of the surface pressure coefficient, $C_{p}$, distributions over the slat, main element, and flap for $\mathrm{Re}=9$ million and $A O A=19^{\circ}$. The calculated distributions agree well with the measurement. The surface pressure coefficients for $\mathrm{AOA}=8^{\circ}$ are presented in Figure 4 . The predicted pressure over the suction side of the the slat is slightly lower than the data. The overall agreement of the predicted surface pressure with the measurement is satisfactory for both cases, showing that the transitional model is capturing the outer "inviscid" flow fields.

Figure 5 shows the calculated and the measured skin friction cocfficients over the main element for $R e=9$ million and $\mathrm{AOA}=19^{\circ}$. The computed results agree very well with the available measured data.

The flow around the slat is complicated and is diffcult to measure due to the physical size of the slat. The transition of the boundary layer from laminar to turbulent flow on the upper suction surface has long been recognized as being one of the critical flow features that are yet to be modeled correctly. In the experiment the onset and the extend of a transition process were determined based upon the skewness, flatness, and standard deviation of hot film data. The transition parameters indicated by the three different measured signals are not always the same and are subject to interpretation. Also, these variables are not derivable from the results of the present computational methodology.

In this study, the onset location of flow transition is assumed to be indicated by the minimum value of the calculated $C_{f}$. Figure 6 shows the calculated $C_{f}$ over the slat for $\mathrm{Re}=9$ million and $A O A=19^{\circ}$. The $C_{f}$ obtained by using the parent turbulence model is also included for comparison.

Figure 7 shows a comparison of the predicted and the measured transition onset locations on the suction side of the three elements for $\mathrm{Re}=9$ million and $\mathrm{AOA}=$ $8^{\circ}, 10^{\circ}, 12^{\circ}, 16^{\circ}$, and $19^{\circ}$. The present model gives rather satisfactory predictions of the transition locations on all three elements for the five AOA measured at this Reynolds number.

The variations of the transition onset locations with AOA on the suction surface of the individual element are shown in Figure 8 . Figure 8 .a shows the slat results. The range of $x / c$ shown corresponds to the slat chord length. The measured data indicated a change of transition location toward the leading edge of the slat as the AOA increases. It is apparent that the present model has predicted this trend well. Moreover, the predicted transition locations also agree well with the measured data. Figures 8.b and 8.c show comparisons between the predicted and the measured transition locations for the main element and flap, respectively. Similar to Figure 8.a, the range of $x / c$ shown correspond to their respective chord lengths. The predicted locations of transition onset on the main element and flap move little with the change of AOA, which agree with the measurement. The predicted onsets occur slightly upstream of the measured locations for both the main and flap.

Another view of the transition locations is given in Figure 9, which shows the locations of the predicted and the measured transition onset on the $30 \mathrm{P} 30 \mathrm{~N}$ airfoil for $\mathrm{AOA}=19^{\circ}$.

Figure 10 shows a comparison of the predicted and the measured transition onset locations on the suction side of the three elements for $\mathrm{Re}=5$ million and $\mathrm{AOA}=$ $8^{\circ}, 10^{\circ}, 12^{\circ}, 16^{\circ}$, and $19^{\circ}$. For this lower Reynolds number, the present model also predicts onset locations that are in a good agreement with the measured data for all three elements.

Figure 11 shows the variation of the transition onset location with AOA for the individual element. Except for the lowest $A O A$ of $8^{\circ}$, the present model predicts reasonably well the transition locations on the suction side of the slat. The flap transition locations were also satisfactorily predicted.

Note that the present transition model predicts the transition location, rather than using it as an input. Through the ranges of angle of attack and Reynolds number tested, the present model has predicted satisfactorily the trend of change of the transition location with angle of attack on all three elements of the 30P30N geometry. The absolute locations of transition onset are also predicted in a fairly consistent manner.

The present transition model uses the evolution of 
the internal peak of the calculated disturbance kinetic energy and the variation of the disturbance in the outer stream to characterize the development of a transitional flow. For a multi-element airfoil, such as the $30 \mathrm{P} 30 \mathrm{~N}$ airfoil, the wakes of the preceding elements convect downstream and further interact with the transitional flow over the succeeding elements. Figure 12 shows the calculated distributions of the disturbance kinetic energy, $k$, along the direction normal to the upper surface of the slat, main, and flap element, respectively, for $\mathrm{Re}=9$ million and $A O A=19^{\circ}$. The local high values in the outer stream represent the high disturbance energy associated with the slat and main wakes. The wakes generated by the preceding elements can also be clearly observed in the velocity magnitude contour plot shown in Figure 13. For this operating condition, the slat wake can be identified as far downstream as in the flap region with little diffusion. It can be argued that there are receptive mechanisms for the boundary layer on the main, or flap, element to be perturbed by the high-intensity disturbances in the slat, or main, wake. In this study, the effect of the wake generated by the preceding element(s) is taken into account by using the highest $k$ in the wake(s) as the $k_{e}$ in Eq.(4) for the succeeding element(s). Near the leading edge of the main element, where the surface normal intersects with the slat cove, $k_{e}$ can vary significantly due to the separated shear layer in the cove region. While not affecting the wall pressure, it causes low amplitude variation in the skin friction and greatly delay convergence locally. In this application, an average value of $k_{e}$ over the intersect region was used for both the main element and the flap.

Figure 14 shows the velocity profiles on the main element and on the flap for $R e=9$ million and $A O A=19^{\circ}$. At $x / c=0.1075$, there is almost an uniform offset between the data and the predictions. This may have been caused by possible improper data calibration $^{2}$. The predicted slat wake is located near the measured wake immediately behind the slat trailing edge $(x / c=0.1075)$. The calculated values for the wake deficit agree reasonably well with the data at $x / c=0.1075$ and 0.45 . The measured wake is wider at $x / c=0.45$. The merging of the calculated slat wake and the main boundary layer is apparently yet to occur at $x / c=0.85$. The boundary layer velocity profiles are predicted well by the present transition model, which results in a better prediction of the main wake immediately behind the main element trailing edge (Figure 14.d, $x / c=0.89817)$.

Over the flap (Figures 14.e and 14.f, $x / c=1.0321$ and 1.1125 ), the predicted development of the slat wake is satisfactory. The predicted deficit of the main element wake is higher than the measured values. 'The width of the calculated main element wake agrees reasonably well with the data.

Figure 15 shows the velocity profiles on the main element for $\mathrm{Re}=9$ million and $\mathrm{AOA}=8^{\circ}$. The predicted main element surface boundary layer is more developed than what the data has shown at $x / c=0.10765$. There is a good agreement between the measurement and the calculated velocity profiles at $x / c=0.45$ and 0.85 , where both the prediction and the measurement show a confluent wake/boundary layer.

Overall, the predicted velocity profiles agree reasonably well with the measurement.

\section{CONCLUDING REMARKS}

The transitional flows over a two-dimensional multi-element airfoil have been computed using a predictive transition model. The model couples an intermittency function correction to a parent two-equation turbulence model through an effective eddy-viscosity for the transitional flow. Comparisons with available measured data for two Reynolds numbers and a range of angle of attack were made. These include the surface pressure, skin friction, and velocity profile. The predicted transition onset on all the elements were also compared with the experimental data. The comparisons show that the present transition model predicts fairly well the locations of transition onset for the cases tested.

\section{ACKNOWLEDGMENT}

This work has been supported by NASA Langley Research Center under a grant NAG 1-1979. The technical monitor is Dr. C.L. Rumsey. The authors would like to thank Dr. S.E. Rogers at NASA Ames Research Center for assistance and advises in using the INS2D and OVERMAGG codes. Dr. C. L. Rumsey and Dr. A. Bertelrud at NASA Langley Research Center kindly provided the experimental data.

\section{REFERENCES}

1 A. Bertelrud, "Transition on a three-element high lift configuration at high Reynolds numbers," AIAA paper 98-0703 (1998).

2 C.L. Rumsey, T.B. Gatski, S.X. Ying, and A. Bertelrud, "Prediction of high-lift flows using 
turbulent closure models," AIAA paper 97-2260 (1997).

${ }^{3}$ S.E. Rogers, F.R. Menter, P.A. Durbin, N.N. Mansour, "A comparison of turbulence models in computing multi-element airfoil flows," AIAA paper 940291 (1994).

4 Bertelrud, A., "Use of empirical transition correlations for flow around high-lift configurations," AIAA paper 99-0541 (1999).

5 W.W.Liou and T.-H. Shih, "Bypass transitional flow calculations using a Navier-Stokes solver and two-equation models," AIAA paper 97-2738 (1997).

6 S.E. Rogers and D. Kwak, "An upwind differencing scheme for the steady-state incompressible NavierStokes equation", Journal of Applied Numerical Mathematics, 8, 43-64, (1991).

7 S.E. Rogers, S.E., D. Kwak, and C. Kiris, "Numerical solution of the incompressible Navier-Stokes equations for steady-state and time-dependent problems," AIAA Journal, 29, 603-610 (1991).

8 S.E. Rogers, "A comparison of implicit schemes for the incompressible Navier-Stokes equations with artificial compressibility," AIAA paper 95-0567 (1995).

9 S.E. Rogers, H.V. Cao, and T.Y. Su, "Grid generation for complex high-lift configurations," AlAA paper 98-3011 (1998).

10 T.-H. Shih and J.L. Lumley, "Kolmogorov behavior of near-wall turbulence and its application in turbulence modeling," Comp. Fluid Dyn., 1, 43-56 (1993).

11 W.W. Liou and T.-H. Shih, "Transonic turbulent flow predictions with new two-equation turbulence models", AIAA paper 95-1805 (1995).

12 W.W. Liou, P.G. Huang, and T.-H. Shih, "Turbulence model assessment for shock wave/turbulent boundary-layer interaction in transonic and supersonic flows," Computers and Fluids, 29, 275-299 (2000).

13 P.C. Stainback, R.J. McGhee, W.D. Beasley, and H.L. Jr. Morgan, "The Langley Research Center's Low Turbulence Pressure Tunnel," AIAA paper 860762(1986). 


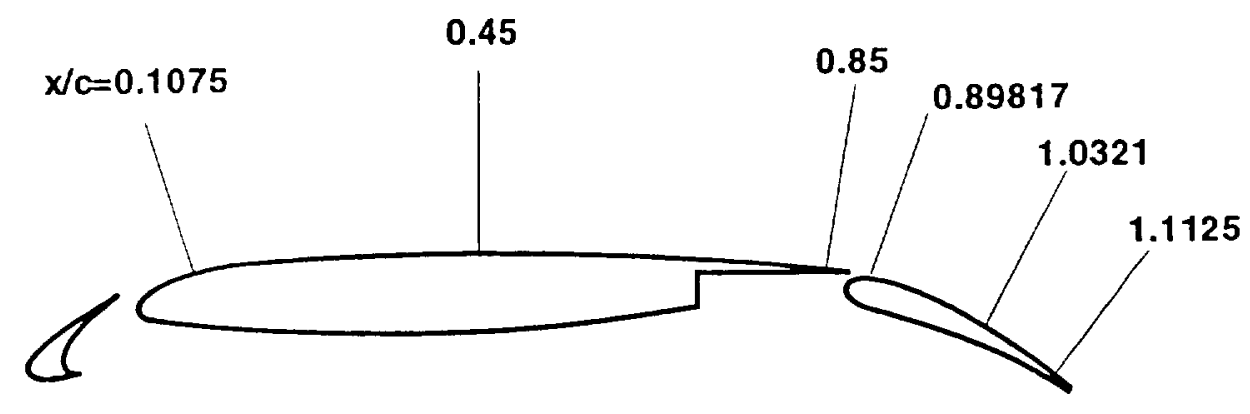

Figure 1. 30P30N geometry and survey station.
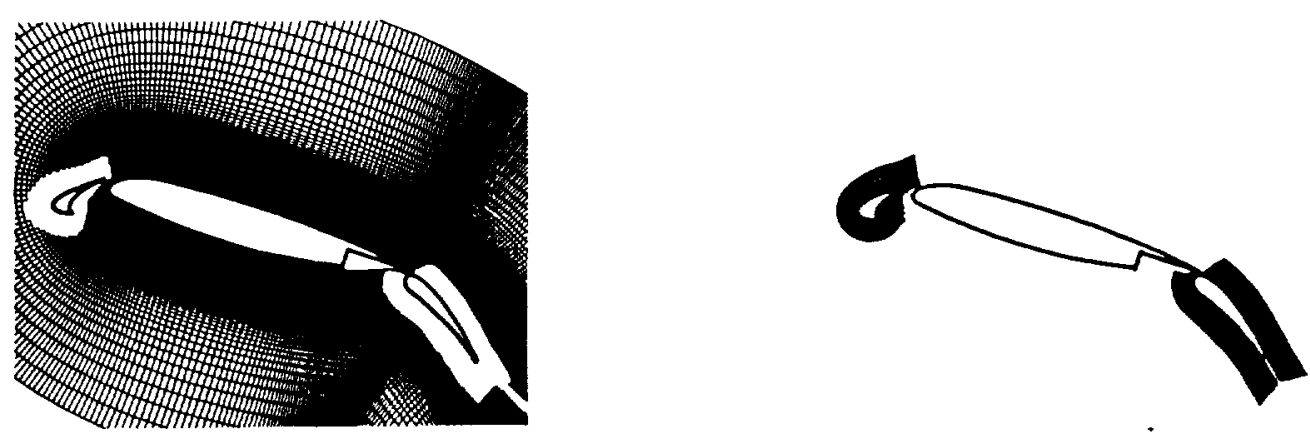

Figure 2. Computational grids.

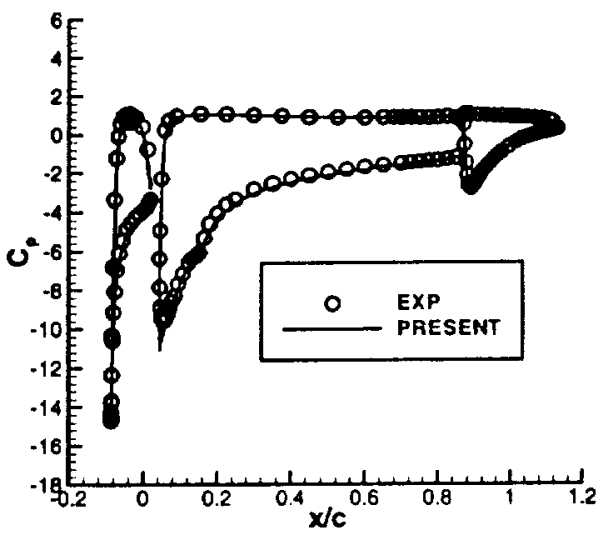

Figure 3. Wall pressure coefficient.

$$
\operatorname{Re}=9 \times 10^{6}, A O A=19^{\circ} \text {. }
$$

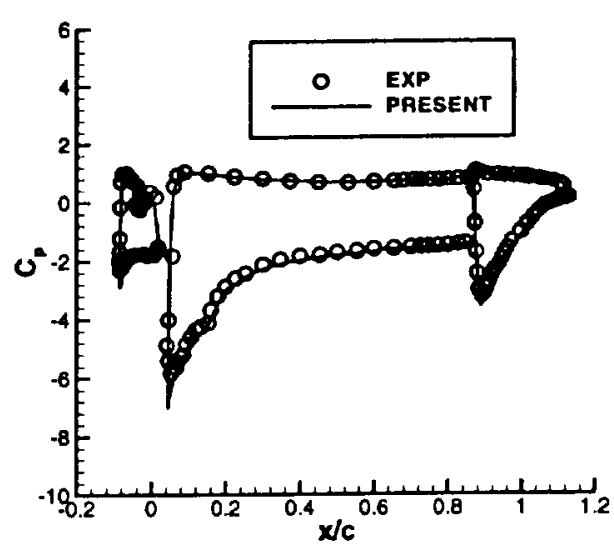

Figure 4. Wall pressure coefficient. $\operatorname{Re}=9 \times 10^{6}, A O A=8^{\circ}$. 


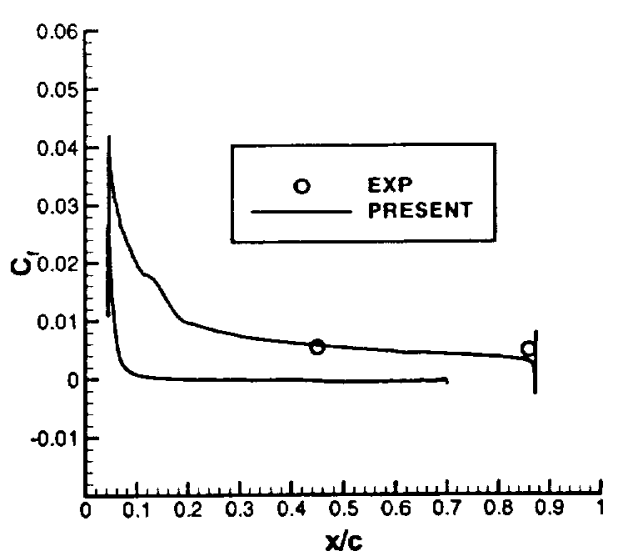

Figure 5. Skin friction coefficient.

$$
\operatorname{Re}=9 \times 10^{6}, A O A=19^{\circ} \text {. }
$$

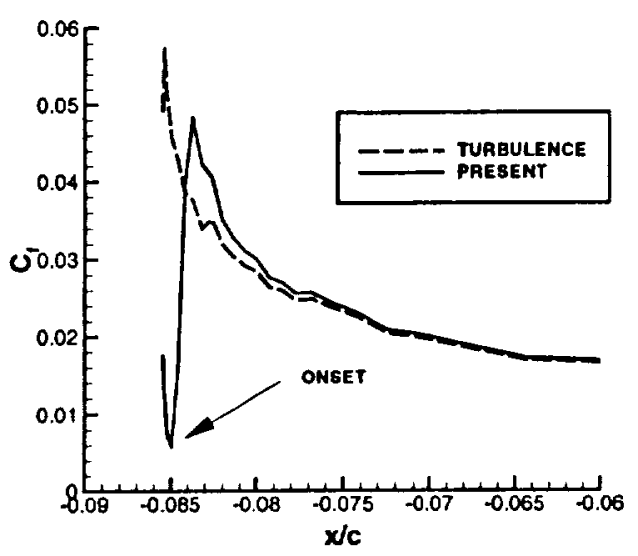

Figure 6. Slat skin friction coefficient. $\operatorname{Re}=9 \times 10^{6}, A O A=19^{\circ}$.

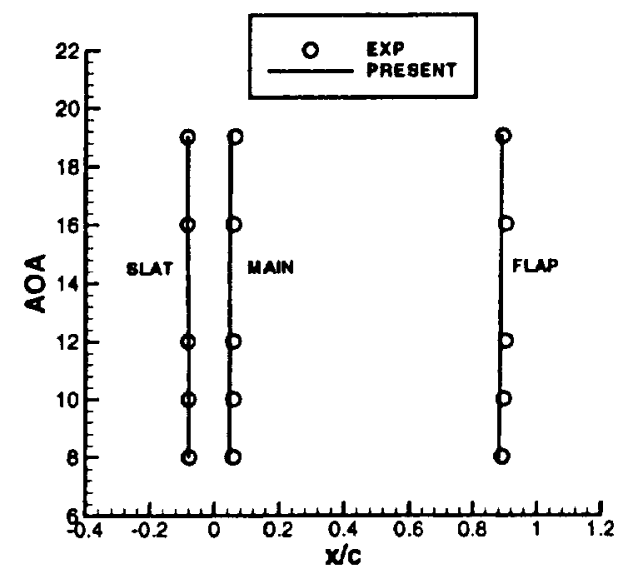

Figure 7. Transition onset. $\operatorname{Re}=9 \times 10^{6}$.

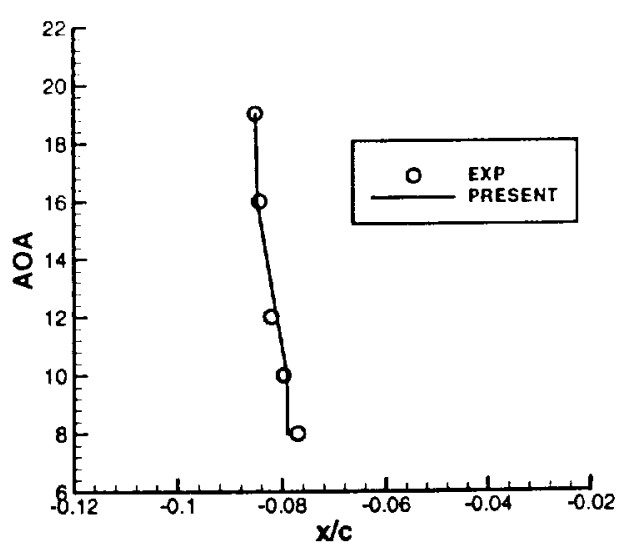

Figure 8.a

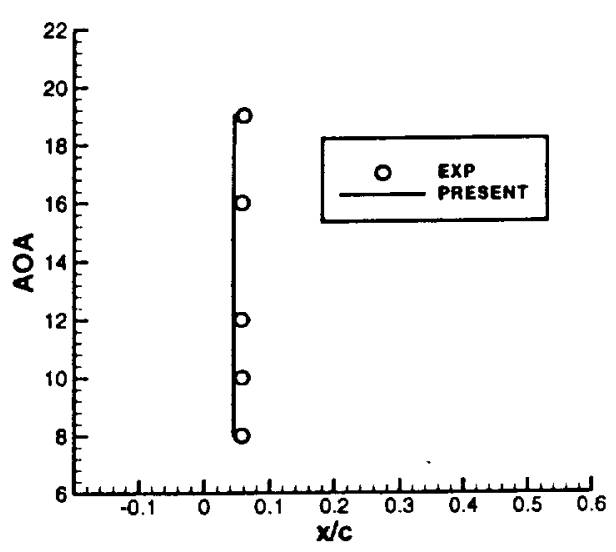

Figure 8.b

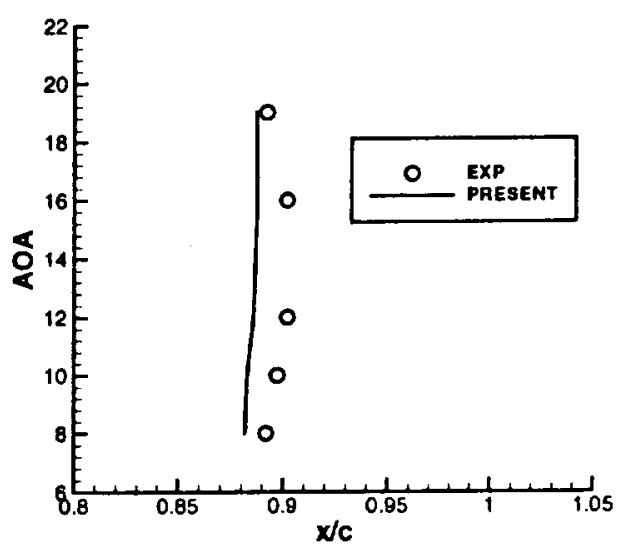

Figure 8.c

Figure 8. Transition onset. $\operatorname{Re}=9 \times 10^{6}$.

(a ) Slat; ( b ) Main; (c ) Flap 



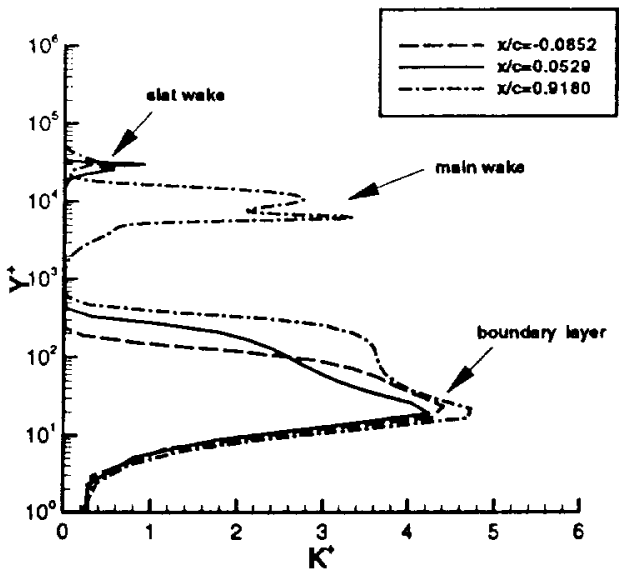

Figure 12. Disturbance kinetic energy. $\operatorname{Re}=9 \times 10^{6}, A O A=19^{\circ}$.

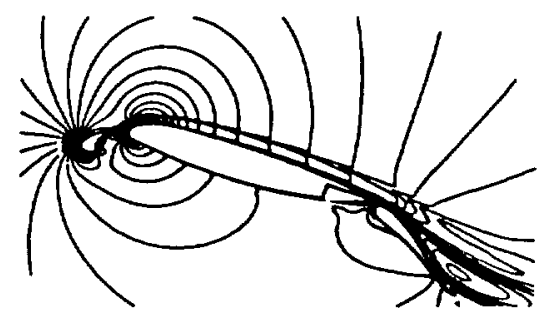

Figure 13. Velocity contour. $\operatorname{Re}=9 \times 10^{6}, \mathrm{AOA}=19^{\circ}$.

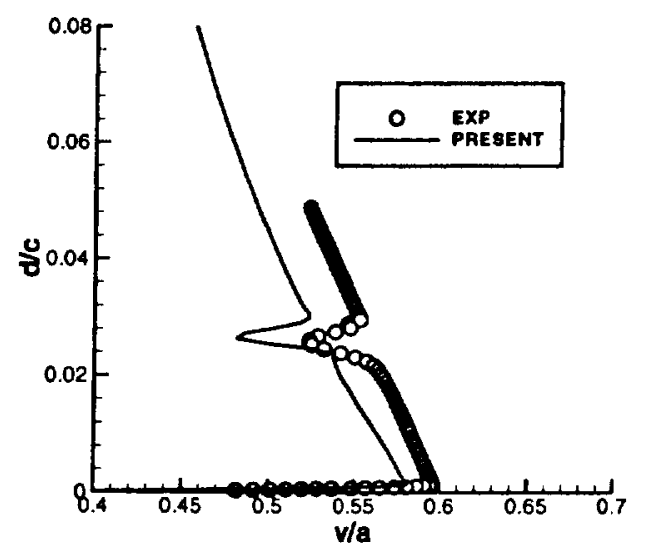

Figure 14.a

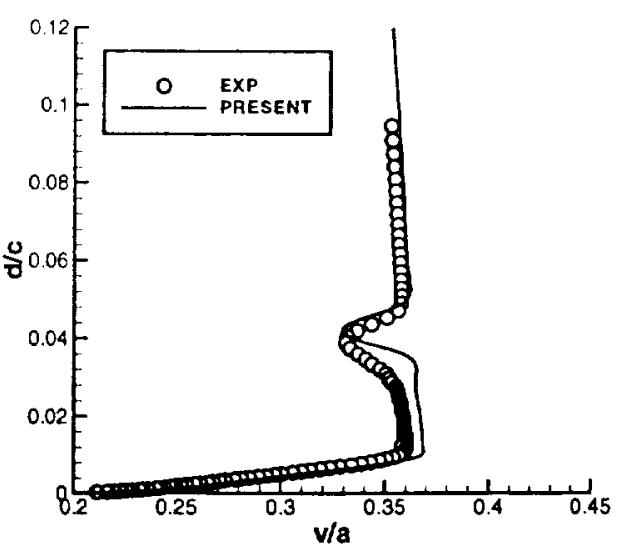

Figure 14.b

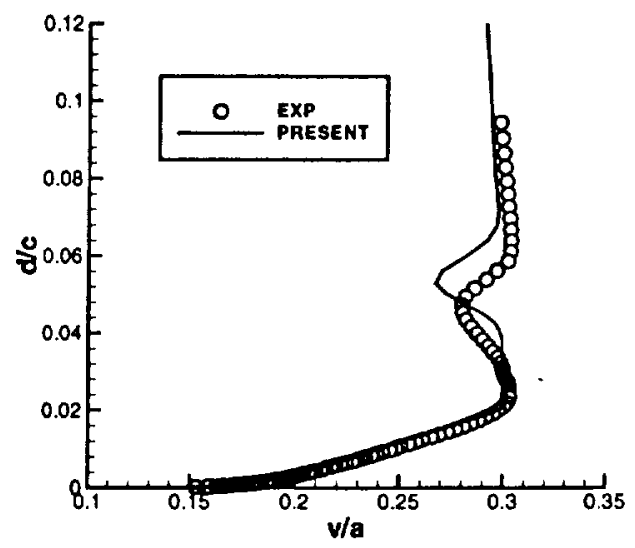

Figure 14.c

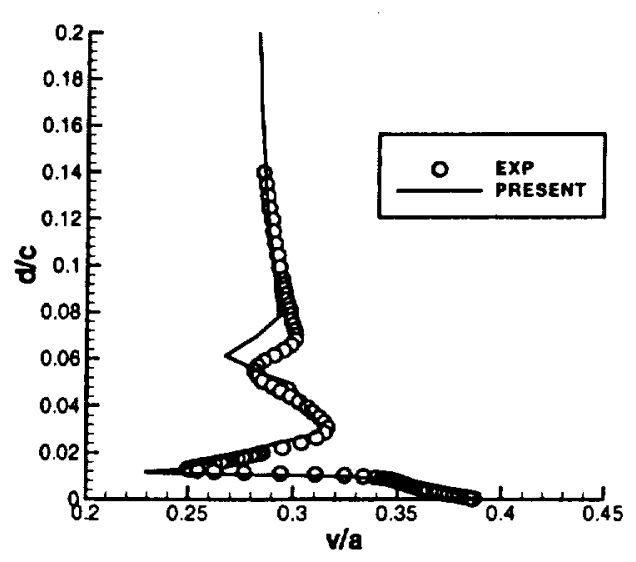

Figure 14.d 


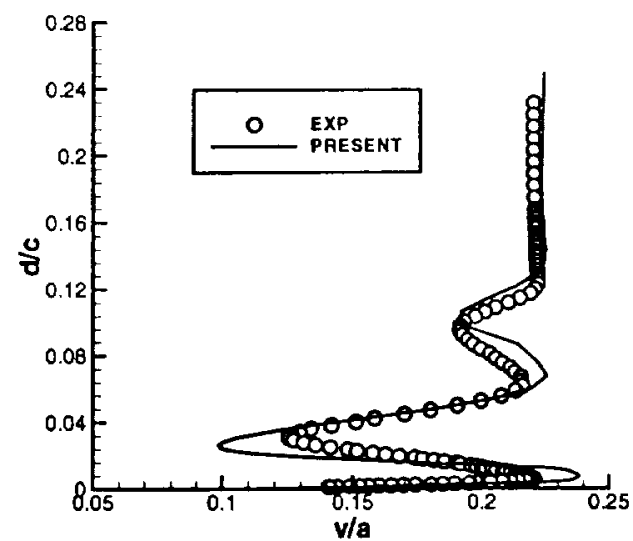

Figure 14.e

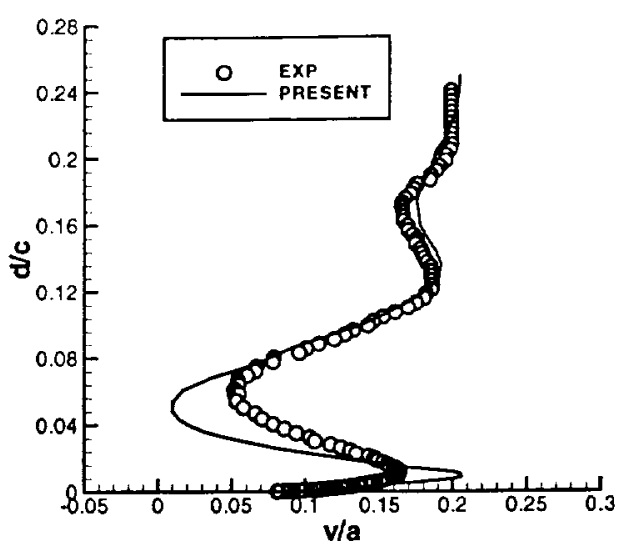

Figure 14.f

Figure 14. Velocity profile. $\operatorname{Re}=9 \times 10^{6}, \mathrm{AOA}=19^{\circ}$. ( a ) $\mathrm{x} / \mathrm{c}=0.1075$ (Main); ( b ) $\mathrm{x} / \mathrm{c}=0.45$ (Main); (c) $x / c=0.85$ (Main); (d ) $x / c=0.89817$ (Flap); (e ) $x / c=1.0321$ (Flap); (f ) $x / c=1.1125$ (Flap)

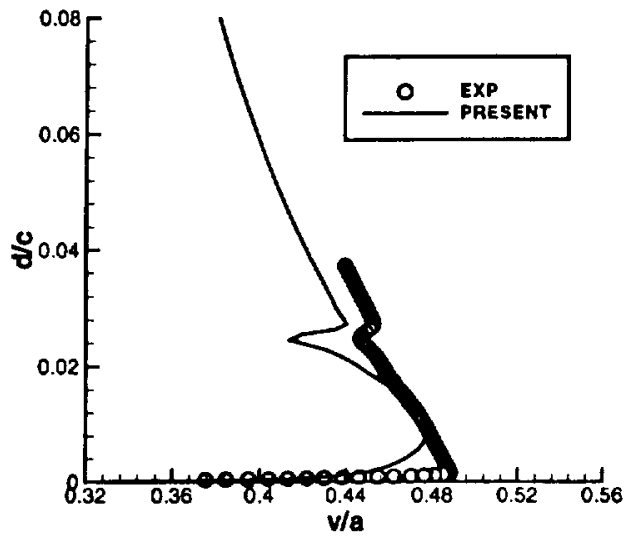

Figure 15.a

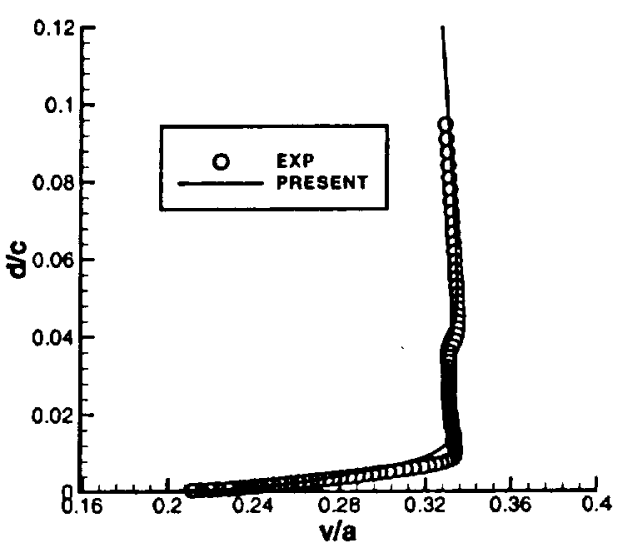

Figure 15.b

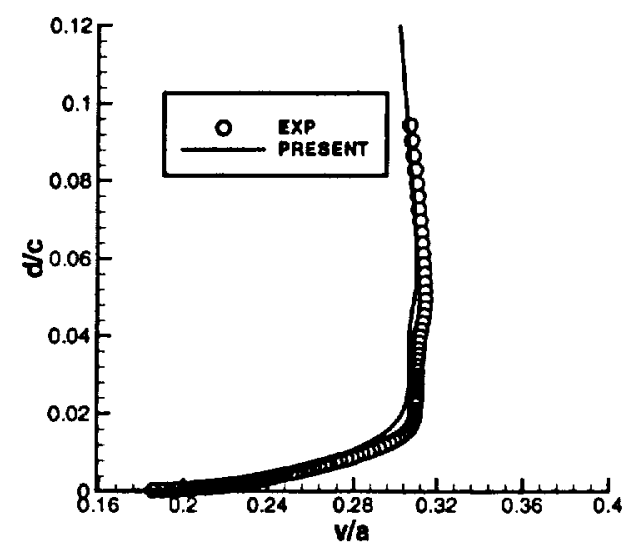

Figure 15.c

Figure 15. Velocity profile. $\operatorname{Re}=9 \times 10^{6}, \mathrm{AOA}=8^{\circ}$. ( a ) $\mathrm{x} / \mathrm{c}=0.1075$ (Main); (b) $x / c=0.45$ (Main); (c ) $x / c=0.85$ (Main). 